\title{
Role of the oxygen uptake efficiency slope in evaluating exercise tolerance
}

\author{
Reizo Baba, Masami Nagashima, Yoshiko Nagano, Masanobu Ikoma, Kenji Nishibata
}

\begin{abstract}
Objective-To investigate the interprotocol agreement of oxygen uptake efficiency slope (OUES).

Methods-16 Japanese children and adolescents ( 10 boys and six girls) underwent two sessions of maximal exercise testing according to the following two treadmill protocols: the standard Bruce protocol and the rapidly increasing staged (RIS) protocol. Maximal oxygen uptake (VO $\mathbf{m a x})$, the ventilatory anaerobic threshold (VAT), and the OUES were obtained from the gas analysis data. Agreement between the protocols was tested by means of the Bland-Altman method.
\end{abstract}

Results-Interprotocol agreement was excellent for the OUES (limit of agreement, $-18 \%$ to $17 \%$ of the mean value), slightly less good for $\mathrm{VO}_{2} \max$ (limit of agreement, $-20 \%$ to $24 \%$ of the mean value), and poor for the VAT (limit of agreement, $-31 \%$ to $31 \%$ of the mean value).

Conclusion-These results confirm the clinical usefulness of the OUES as a measure of evaluating exercise tolerance in the paediatric population.

(Arch Dis Child 1999;81:73-75)

Keywords: cardiopulmonary exercise testing; maximal oxygen uptake; anaerobic threshold; oxygen uptake efficiency slope

The pattern of physical activities that children usually engage in is characterised by short bursts of high intensity exercise. ${ }^{12}$ Therefore, standard incremental exercise protocols, such as the Bruce protocol, used in most exercise laboratories may not be representative of their

Accepted 9 March 1999

$$
y=1017 x-697
$$
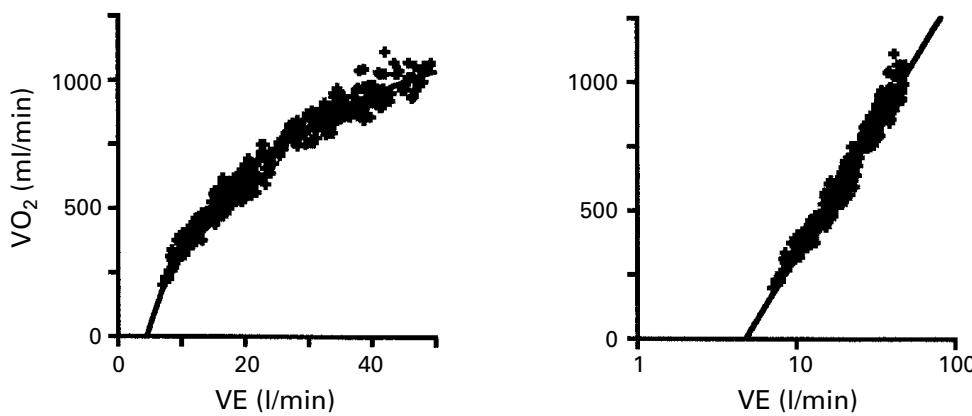

Figure 1 Relation between oxygen uptake $\left(\mathrm{VO}_{2}\right)$ and minute ventilation (VE) during incremental exercise in a representative subject (a 13 year old boy). The constant for the log term of the curve fitting equation (1017 for this subject) is defined as the oxygen uptake efficiency slope (OUES). The data are presented as linear (left) and semilog plots (right) of the $x$ axis. everyday activities, because they take sustained periods of exercise intensity at each increased workload. ${ }^{2}$ For this reason, we have adopted an exercise protocol of shorter duration lasting several minutes or less, called the rapidly increasing staged protocol (the RIS protocol). ${ }^{3}$

The measurement of maximal oxygen uptake $\left(\mathrm{VO}_{2} \mathrm{max}\right)$ is widely used as the standard of cardiopulmonary functional reserve in children and adolescents. ${ }^{4}$ However, exercise protocols can affect the measurement of $\mathrm{VO}_{2} \max .^{6-8}$ Oxygen uptake at the ventilatory anaerobic threshold (VAT) is the most commonly used index of aerobic capacity ${ }^{9-11}$ that does not require the subject to perform maximal exercise. It is a gas exchange measurement that can non-invasively detect the oxygen uptake at the onset of lactate acidosis. Therefore, the VAT can be a useful tool for the evaluation of patients with exercise limitation, including children and adolescents with heart disease. However, studies suggest that the reproducibility of VAT results can also be affected by the exercise protocol used. ${ }^{6712}$

Recently, Baba et al introduced a new index of cardiorespiratory functional reserve, the oxygen uptake efficiency slope (OUES), which is derived from the logarithmic curve fitting model between oxygen uptake $\left(\mathrm{VO}_{2} ; \mathrm{ml} / \mathrm{min}\right)$ and minute ventilation (VE; litres/min) during incremental exercise (fig 1). ${ }^{13-15}$ This index is related to both the pulmonary dead space and metabolic acidosis. ${ }^{13} 14$ One of the greatest advantages of the OUES is that it is not greatly affected by exercise intensity. ${ }^{13}{ }^{14}$ This advantage is important when a progressive exercise test is not completed because the subject is either unwilling or unable to complete the test. Baba et al also reported that OUES correlates strongly with $\mathrm{VO}_{2} \max (r=0.941)$, using a paediatric population consisting of normal subjects and patients with a wide variety of cardiac diseases ${ }^{13}$ suggesting a potential usefulness for this measurement in the evaluation of exercise tolerance in these patients. However, changes in the exercise protocol may affect OUES because $\mathrm{VO}_{2}$ and $\mathrm{VE}$ have different time constants of response. ${ }^{16}{ }^{17}$ Therefore, our objective was to investigate the between protocol (that is, between the Bruce protocol and the RIS protocol) agreement of OUES, in comparison with $\mathrm{VO}_{2}$ max and VAT.

\section{Methods}

The subjects were 16 Japanese children and adolescents ( 10 boys and six girls) who volunteered to participate in our study. Their mean age was 12.7 years (SD, 2.8; range, 8.0-18.3) and mean body weight was $41 \mathrm{~kg}$ (SD, $14 \mathrm{~kg}$; 
Table 1 Mean values of exercise testing and their limits of agreement between two treadmill protocols (Bruce and RIS)

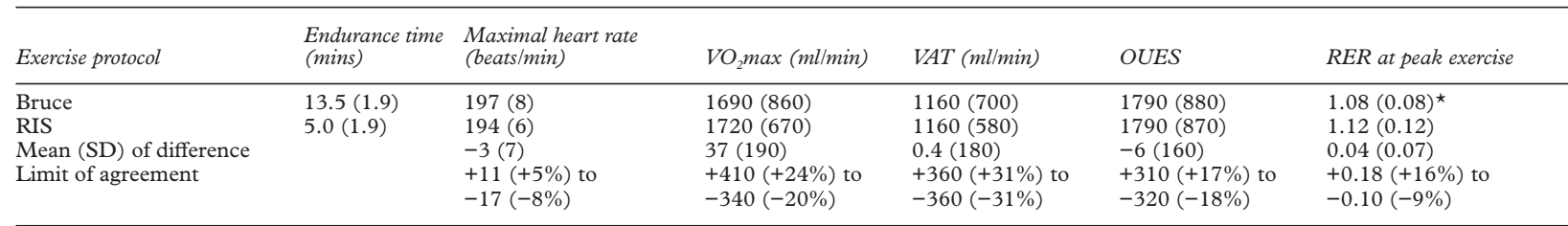

Data are expressed as mean (SD). Limits of agreement are expressed as absolute values and the percentages of the mean values of the two sessions in parentheses. ${ }^{\star} \mathrm{p}<0.05$ between the two protocols.

OUES, oxygen uptake efficiency slope; RER, the respiratory exchange ratio; RIS, rapidly increasing staged protocol; VAT, oxygen uptake at the ventilatory anaerobic threshold; $\mathrm{VO}_{2}$ max, maximal oxygen uptake.

range, $22-73 \mathrm{~kg}$ ). A physician's interviews and physical examinations revealed that they were in good health. All subjects were moderately active, but did not participate in daily exercise events at competitive levels. None of the subjects was a habitual smoker. The subjects and their parent(s) gave written informed consent for participation in our study.

Exercise tests were performed on a treadmill (MAT-2500; Fukuda Denshi Co Ltd, Tokyo, Japan). The subjects underwent two sessions of exercise testing until exhaustion, following two exercise protocols: the standard Bruce protocol and the RIS protocol. ${ }^{3}$ In the RIS protocol, the slope and the speed of each stage were the same as those of the Bruce protocol, but the duration was 15 seconds during the first four stages and three minutes during each subsequent stage. ${ }^{3}$ We adopted the RIS protocol because its intensity and short duration reflect children's exercise mode. The protocols were administered seven days apart, the order of the sessions being assigned randomly. We monitored electrocardiography and heart rate throughout the tests with the stress test system (ML-5000; Fukuda Denshi). We measured cuff blood pressure every minute with an automatic indirect manometer (STBP-680F; Collin Denshi, Nagoya, Japan).

Carbon dioxide production $\left(\mathrm{VCO}_{2} ; \mathrm{ml} / \mathrm{min}\right.$, STPD), $\mathrm{VO}_{2}$ ( $\left.\mathrm{ml} / \mathrm{min}, \mathrm{STPD}\right), \mathrm{VE}(1 / \mathrm{min}$, BTPS), tidal volume (ml, BTPS), respiratory rate (breaths $/ \mathrm{min}$ ), and the mixed expiratory carbon dioxide concentration (\%) were measured continuously on a breath by breath basis with a Minato AE-280 metabolic measurement chart (Minato Medical Science, Osaka, Japan) equipped with an oxygen and carbon dioxide analyser. We measured respiratory flow by the thermal dissipation technique. To calculate $\mathrm{VO}_{2} \mathrm{max}$, we averaged values obtained during the final 15 seconds of exercise. For the determination of VAT, data were processed with a five breath moving average filter to reduce breath by breath "noise". We defined VAT as the value of $\mathrm{VO}_{2}$ at which an increase in $\mathrm{VE} / \mathrm{VO}_{2}$ occurred without a simultaneous increase in $\mathrm{VE} / \mathrm{VCO}_{2}{ }^{9}$ The following equation was used to determine OUES: $\mathrm{VO}_{2}=\mathrm{a} \log _{10} \mathrm{VE}+\mathrm{b}$, where " $\mathrm{a}$ " and "b" were constants, and "a" was OUES. ${ }^{13} 14$

We assessed agreement between measurements obtained from the two test sessions with the limits of agreement by Bland and Altman. ${ }^{18}$ A $p$ value $<0.05$ was considered significant.

\section{Results}

Table 1 summarises the results. All exercise tests were completed without complications. We could determine the VAT in all sessions. There were no between protocol differences in the mean values except the respiratory gas exchange ratio $\left(\mathrm{VCO}_{2} / \mathrm{VO}_{2}\right)$ at peak exercise (table 1). Interprotocol variability was small in the OUES, slightly greater in $\mathrm{VO}_{2} \mathrm{max}$, and large in the VAT (table 1).

\section{Discussion}

Our study was intended to elucidate the influence of the two treadmill protocols, (the Bruce protocol and the RIS protocol) on $\mathrm{VO}_{2} \mathrm{max}$, the VAT, and the OUES. Results show that agreement between the two treadmill protocols was excellent for the OUES. This confirms the clinical usefulness of the OUES for the evaluation of exercise tolerance in children and adolescents.

The pattern of physical activities that children usually engage in differs from that of adults in that their activities are characterised by short bursts of high intensity exercise, not by sustained exercise. ${ }^{12}$ However, evaluation of physiological responses to exercise in children has been based almost exclusively on protocols developed for adults (such as the Bruce protocol) that usually last 10 minutes or more. For this reason, we have adopted the RIS protocol, ${ }^{3}$ an exercise protocol lasting several minutes or less, which we believe is more representative of children's daily activities.

Evaluation of exercise tolerance in children has traditionally been focused on the measurement of $\mathrm{VO}_{2}$ max. ${ }^{245}$ However, a true plateau of $\mathrm{VO}_{2}$ while the work rate continues to increase, which is believed to be the most reliable criterion of maximal exercise in adults, is rarely seen in children and adolescents. ${ }^{2} 101920$ Because of the lack of a clear definition of maximal exercise in these populations, $\mathrm{VO}_{2} \mathrm{max}$ is effort dependent and thus is substantially influenced by the child's motivation, which may, at least in part, account for the slightly larger between test variability of $\mathrm{VO}_{2} \mathrm{max}$. Moreover, in their daily activities, children rarely achieve $\mathrm{VO}_{2} \max$, which can probably be seen only in exercise laboratories. $^{210}$ In addition, past studies reported a large effect of exercise protocol on the $\mathrm{VO}_{2}$ max..$^{6-8}$ Therefore, the use of $\mathrm{VO}_{2} \max$ as the "gold standard" of children's exercise tolerance is questioned. Peak $\mathrm{VO}_{2}$, simply measured at the end of a test, is widely used as a substitute. However, the end point of an exercise test is greatly influenced by motivation 
of the subject, particularly for young children and those with cardiopulmonary disease.

Rather than the measurements obtained from maximal exercise, clinically valuable information on exercise testing may lie in the submaximal phases. ${ }^{2}$ One of the most frequently used submaximal measurements is the VAT, ${ }^{9-11}$ which can be determined noninvasively from the dynamic responses of gas exchange data during incremental exercise testing. Although various results have been reported as to the effect of the exercise protocol on the VAT, our results show that agreement of the VAT between the two treadmill protocols was poor, the limit of agreement being $\pm 30 \%$ of the mean value. Therefore, care must be taken in the interpretation of the results of the VAT as an index of children's exercise tolerance. We have introduced the OUES, which is related to both the pulmonary dead space and metabolic acidosis and is strongly correlated with $\mathrm{VO}_{2} \max (r=0.941)$ in a paediatric population. ${ }^{13}$ One of the greatest advantages of the OUES is that it is not greatly affected by exercise intensity. ${ }^{1314}$ Moreover, in contrast to the VAT, in which the measurement is substantially influenced by the changes in the evaluator, ${ }^{12}$ OUES is free from inter-evaluator variability, because it is mathematically determined by a set of gas analysis data. The results of our study suggesting that the OUES is virtually independent of treadmill protocol adds to the information about the usefulness of this measurement for the evaluation of exercise tolerance in the paediatric population.

1 Bailey RC, Olson J, Pepper SL, Barstow TJ, Porszsasz J, Cooper DM. The level and tempo of children's physical activities: an observational study. Med Sci Sports Exerc 1995;27:1033-41.

2 Cooper DM. Rethinking exercise testing in children: a challenge. Am $\mathcal{F}$ Respir Crit Care Med 1995;152:1154-7.
3 Nagashima M, Baba R, Goto M, Nishibata K, Nagano Y. Exercise-induced ventricular tachycardia without demontrable heart disease in childhood. Acta Paediatr fpn 1996; 38:495-9.

4 Day L. The testing, prediction and significance of maximal aerobic power in children. Australian fournal of Sports Sciences 1981;1:18-22.

5 Mayers N, Gutin B. Physiological characteristics of elite prepubertal cross country runners. Med Sci Sports 1979;11: $172-6$.

6 Buchfuhrer MJ, Hansen JE, Robinson TE, Sue DY, Wassemann K, Whipp BJ. Optimizing the exercise protocol for cardiopulmonary assessment. F Appl Physiol 1983;55: 1558-64.

7 Davis JA, Vodak P, Wimore JH, Vodak J, Kurtz P. Anaerobic threshold and maximal aerobic power for the three modes of exercise. 7 Appl Physiol 1976;41:544-50.

8 Hermansen L, Saltin B. Oxygen uptake during maximal treadmill and bicycle exercise. $\mathcal{F}$ Appl Physiol 1969;26:31-7.

9 Wassermann K, Whipp BJ, Koyal SN, Beaver WL. Anaerobic threshold and respiratory gas exchange during exercise. bic threshold and respiratory gas

10 Cooper DM, Weiler-Ravell D, Whipp BJ, Wasserman K. Aerobic parameters of exercise as a function of body size during growth in children. $\mathcal{F}$ Appl Physiol 1984;56:628-34.

11 Reybrouck T, Weymans M, Stijns H, Knops J, van der Hauwaert HL. Ventilatory anaerobic threshold in healthy children: age and sex differences. Eur F Appl Physiol 1985; 54:278-84.

12 Shimizu M, Myers J, Buchanan N, et al. The ventilatory threshold: method, protocol, and evaluator agreement. Am Heart $\mathcal{F}$ 1991;122:509-16.

13 Baba R, Nagashima M, Goto $M$, et al. Oxygen uptake efficiency slope: a new index of cardiorespiratory functional reserve derived from the relation between oxygen uptake and minute ventilation during incremental exercise. $\mathfrak{f} \mathrm{Am}$ Coll Cardiol 1996;28:1567-72.

14 Baba R, Tsuyuki K, Kimura Y, et al. Oxygen uptake efficiency slope as a useful measure of cardiorespiratory functional reserve in adult cardiac patients. Eur $\mathcal{f} A p p l$ Physiol. [In press.]

15 Baba R, Kubo N, Morotome Y, Iwagaki S. Reproducibility of the oxygen uptake efficiency slope in normal healthy subjects. $\mathcal{F}$ Sport Med Phys Fit. [In press.]

6 Casaburi R, Barstow TJ, Robinson T, Wasserman K. Influence of work rate on ventilatory and gas exchange kinetics. 7 Appl Physiol 1989;67:547-55

17 Whipp BJ, Ward SA, Lamarra NL, Davis JA, Wasserman K. Parameters of ventilatory gas exchange dynamics during exercise. F Appl Physiol 1982;52:1506-13.

18 Bland JM, Altman DG. Statistical method for assessing agreement between two methods of clinical measurement. Lancet 1986;i:307-10.

19 Cunningham DA, MacFalane B, Paterson DH, Lefcoe M, Sangal SD. Reliability and reproducibility of maximal oxygen uptake measurements in children. Med Sci Sports gen uptake mea

20 Rowland TW. Does peak $\mathrm{VO}_{2}$ reflect $\mathrm{VO}_{2} \mathrm{max}$ in children? Evidence from supermaximal testing. Med Sci Sports Exerc 1992;25:689-93. 